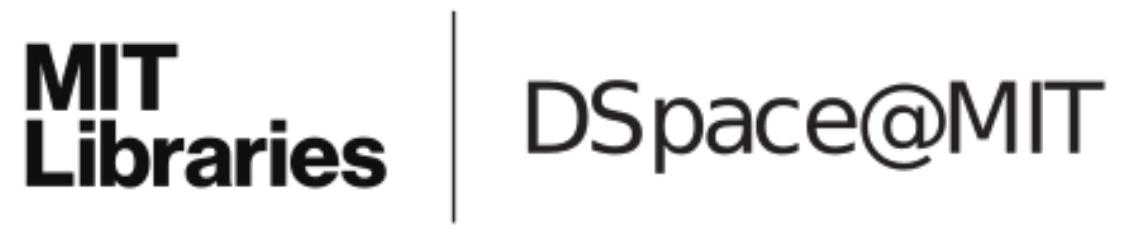

\author{
MIT Open Access Articles
}

Mechanism for recombination of radiation-
induced point defects at interphase boundaries

The MIT Faculty has made this article openly available. Please share how this access benefits you. Your story matters.

Citation: Liu, X.-Y. et al. “Mechanism for Recombination of Radiation-induced Point Defects at Interphase Boundaries." Physical Review B 85.1 (2012): 012103. (C) 2012 American Physical Society.

As Published: http://dx.doi.org/10.1103/PhysRevB.85.012103

Publisher: American Physical Society

Persistent URL: http://hdl.handle.net/1721.1/72459

Version: Final published version: final published article, as it appeared in a journal, conference proceedings, or other formally published context

Terms of Use: Article is made available in accordance with the publisher's policy and may be subject to US copyright law. Please refer to the publisher's site for terms of use. 


\title{
Mechanism for recombination of radiation-induced point defects at interphase boundaries
}

\author{
X.-Y. Liu, ${ }^{1}$ B. P. Uberuaga, ${ }^{1}$ M. J. Demkowicz, ${ }^{2}$ T. C. Germann, ${ }^{1}$ A. Misra, ${ }^{1}$ and M. Nastasi ${ }^{1}$ \\ ${ }^{1}$ Los Alamos National Laboratory, Los Alamos, New Mexico 87545, USA \\ ${ }^{2}$ Department of Materials Science and Engineering, Massachusetts Institute of Technology, Cambridge, Massachusetts 02139, USA
}

(Received 28 October 2011; published 13 January 2012)

\begin{abstract}
Interfaces play a critical role in the extraordinary resistance to irradiation damage in nanostructured materials. Atomistic simulations are performed to examine defect production and recovery at incoherent interphase boundaries with different atomic structures. The interstitials produced during cascades and absorbed by the interface are subsequently observed to emit from the interface to annihilate residual vacancies in the nearby bulk. These results indicate that interstitials do not "lose their identity" when absorbed at interfaces regardless of the extent of delocalization at boundaries.
\end{abstract}

DOI: 10.1103/PhysRevB.85.012103

PACS number(s): 68.35.-p, 61.80.Az, 61.82.Rx, 68.65.-k

Developing advanced nuclear energy systems depends crucially on the improved understanding of radiation-damage processes in reactor materials. It is clear that the requirement for significantly higher radiation tolerance materials poses great challenges to currently available materials. ${ }^{1,2}$ Nanostructured ferritic alloy materials show great promise of high performance and radiation-damage tolerance. ${ }^{3,4}$ Such nanocomposite materials have extremely high interphase boundary area per unit volume as compared to monolithic structural metals and their enhanced radiation-damage tolerance is presumably a consequence of efficient point defect recovery at interphase boundaries. ${ }^{3,4}$ During collision cascades, boundaries absorb interstitials, leaving behind vacancies in the bulk, ${ }^{5,6}$ yet it is not clear how subsequent defect recombination is enabled by boundaries.

Recently, an interstitial emission mechanism was proposed for high-symmetry, special grain boundaries in single-phase metals $^{7}$ where the interstitials with compact cores were observed to reemit from the grain boundary to recombine with vacancies near the boundaries. This type of self-healing mechanism may render nanomaterials highly useful for nuclear applications. ${ }^{8}$ However, for single-element materials, grain boundaries would be subjected to rapid grain growth during irradiation and, therefore, the effectiveness of this mechanism may be lost. For immiscible composites, the nanoscale length scales in the as-synthesized material may be retained during irradiation. Furthermore, in semi- or incoherent interphase boundaries, the interstitial core may delocalize in the boundary plane. ${ }^{9}$ With a delocalized displacement field around an interstitial, the interstitial may effectively "lose its identity" in the interphase boundary and may not be able to reemit. Thus, the atomic scale mechanisms that enable defect recombination at interphase boundaries of different atomic structures need to be understood to allow mechanistically informed interface design for optimized nanocomposite materials that can operate at extreme radiation environments.

In this Brief Report, we describe atomistic modeling studies of defect-interface interactions, as they relate to radiation environments, at a fcc-bcc interface, namely, $\mathrm{Cu}-\mathrm{Nb}$, as a model system since experimentally nanolayered fcc-bcc composites such as $\mathrm{Cu}-\mathrm{Nb}$ (Refs. 10 and 11) exhibit significantly enhanced radiation-damage tolerance as compared to monolithic metals. Also, accurate interatomic potentials are available that predict a variety of atomic arrangements with nearly degenerate energies and varying levels of point defect localization. Thus, the defect recombination behavior can be explored for a range of interface structures to develop generalized mechanisms. There is indeed a lot of work connect radiation-damage response in materials to the bulk properties such as defect formation and migration energies, and defect structures (for example, one-dimensional vs three-dimensional interstitials). ${ }^{12}$ This paper is to examine other aspects of the specific interaction of defects with interfaces and the role of the interfacial structure.

Two different types of interfaces were studied. The Kurdjumov-Sachs (KS) interface, ${ }^{13}$ which was chosen as it has been extensively investigated both experimentally ${ }^{14}$ and theoretically, ${ }^{9,15,16}$ and follows the traditionally observed KS orientation relation (OR): $\{111\}_{\mathrm{fcc}} \|\{110\}_{\mathrm{bcc}}$, with $\langle 110\rangle_{\text {fcc }} \|\langle 111\rangle_{\text {bcc }}$ at the interface. The other type of interface also forms in the KS OR, but differs in the orientation of the habit plane, $\{112\}_{\mathrm{fcc}} \|\{112\}_{\mathrm{bcc}}$, while retaining $\langle 110\rangle_{\text {fcc }} \|\langle 111\rangle_{\text {bcc }}$ at the interface, for convenience sake termed $\{112\}$ interface. This interface was recently found in severely plastic deformed (SPD) fcc-bcc composites. ${ }^{17}$ One important difference between these two interfaces is that in the KS interfaces, point defects are strongly delocalized (with core size of defects approximately $3 \mathrm{~nm}$ in diameter) while in the $\{112\}$ interface, point defects absorbed remain compact. ${ }^{18}$

We use embedded-atom method (EAM $)^{19}$ interatomic potentials for the $\mathrm{Cu}-\mathrm{Nb}$ system. ${ }^{20}$ To construct the KS bilayer interface model, an unrelaxed (111) surface slab of perfect $\mathrm{Cu}$ crystal is joined together with an unrelaxed (110) surface slab of $\mathrm{Nb}$ crystal, in the interface OR defined above. The translation of the adjoining $\mathrm{Cu}$ and $\mathrm{Nb}$ layers with respect to each other as rigid bodies was considered during the relaxation process. Two KS interface structures were considered in this study: $\mathrm{KS}_{1}$ and $\mathrm{KS}_{\min }$. $\mathrm{KS}_{\min }$ has $5 \%$ vacancies in the $\mathrm{Cu}$ layer adjacent to the interface compared to $\mathrm{KS}_{1}$ and a slightly lower interfacial energy. Both structures have been studied extensively. ${ }^{9}, 15,16,20-22$ A similar procedure was applied to construct the $\{112\}$ interface model. ${ }^{18}$

We begin with molecular dynamics (MD) studies of collision cascades in a bilayer model of the $\mathrm{KS}_{1}$ interface, to understand the role of the interface on defect production during radiation-damage events. The bilayer simulation cell 


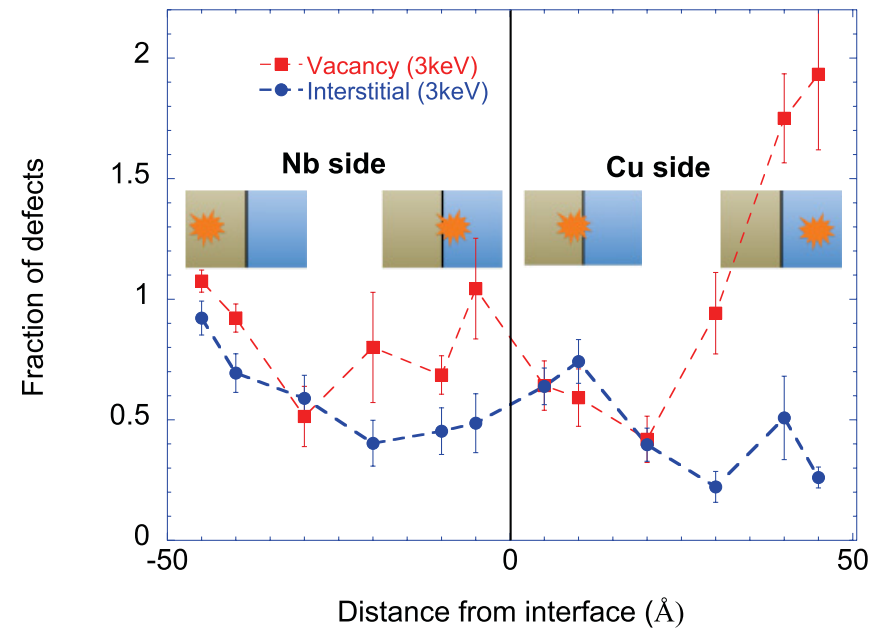

FIG. 1. (Color online) The statistically averaged fraction of defects produced from $3 \mathrm{keV}$ cascades after $60 \mathrm{ps}$, as a function of PKA distance from $\mathrm{KS}_{1}$ interface, on the left- $\mathrm{Nb}$ side, and on the right $-\mathrm{Cu}$ side. The fraction of defects is defined as $F_{\text {defects }}=n_{\mathrm{Cu}} / n_{\mathrm{Cu}}^{\text {bulk }}+n_{\mathrm{Nb}} / n_{\mathrm{Nb}}^{\text {bulk }}$, where $n_{i}$ is the radiation-induced defect number in bilayers, while $n_{i}^{\text {bulk }}$ is the defect number in single crystal for the $i$ th species. In the insets, the locations of primary cascade damage are marked.

is $13.8 \times 15.7 \times 9.8 \mathrm{~nm}$ in $x, y, z$ directions, with the interface plane in $x z$, containing 148484 atoms. The crystallographic directions of $x, y$, and $z$ are $[11 \overline{2}]_{\mathrm{fcc}} \|[1 \overline{1} 2]_{\mathrm{bcc}}$ in $x$, $[111]_{\mathrm{fcc}} \|[110]_{\mathrm{bcc}}$ in $y$, and $[1 \overline{1} 0]_{\mathrm{fcc}} \|[\overline{1} 11]_{\mathrm{bcc}}$ in $z$. The system was initially thermally equilibrated at $300 \mathrm{~K}$ for $10 \mathrm{ps}$ before a primary knock-on (PKA) atom of $3.0 \mathrm{keV}$ was introduced at varying distances $(0.5-4.5 \mathrm{~nm})$ away from and directed toward the interface. In a typical collision cascade, the initial thermal spike cools rapidly after a few picoseconds. The simulations were continued for an additional $60 \mathrm{ps}$ to reach a stable defect configuration. The surviving defects, excluding those absorbed into the interface itself, were then counted through a reference mapping analysis. ${ }^{7}$ For each simulation condition, ten MD simulations with different random number seeds were performed to generate a statistical average.

The statistically averaged amount of defects produced, relative to that produced in equivalent cascades in the single crystal, is shown as a function of PKA distance in Fig. 1. Beginning with PKAs on the $\mathrm{Cu}$ side of the interface, when the PKA is initiated far from interface, the produced defects reside mainly in the $\mathrm{Cu}$ layer. The MD simulations show that almost all interstitials surviving upon subsequent annealing after the collision cascade are absorbed into the interface due to both the thermodynamic driving force of the interface acting as a sink for defects and the high mobility of interstitials in bulk $\mathrm{Cu}\left(\sim 0.1 \mathrm{eV}\right.$ migration energy $\left.{ }^{9,15}\right)$. On the other hand, the concentration of vacancies produced is up to twice that produced in equivalent cascades produced in single-crystal $\mathrm{Cu}$. As the PKA-interface distance is reduced, however, a growing fraction of the cascade damage resides in the $\mathrm{Nb}$ layer. As the cascades interact more strongly with the interface and the $\mathrm{Nb}$ layer, we observe a diminishing difference between the interstitial and vacancy defects produced in the cascade.
Interestingly, Fig. 1 also reveals that the trend in defect production as a function of PKA distance from the $\mathrm{Nb}$ side is reversed compared to the $\mathrm{Cu}$ side: away from the interface, the vacancy and interstitial numbers are nearly equal, while closer to the interface there is a growing difference between the numbers of each type of defects produced. These trends demonstrate that when the cascade interacts with the $\mathrm{Cu}$ layer, $\mathrm{Cu}$ interstitials are created which can be loaded into the interface. However, $\mathrm{Nb}$ defects do not load significantly into the interface during the cascades.

Thus, on the short time scales of collision cascades, interstitials are preferentially loaded into the interface while vacancies remain trapped in the bulk. To understand how these loaded interstitials subsequently interact with the residual vacancies on longer time scales, we preload $\mathrm{Cu}$ interstitials to the interface and determine the effect on the formation energy of vacancies. The number of interstitials introduced into the interface corresponds to an effective area density of interstitials of about $1 / 60 \AA^{2}$.

In Figs. 2(a)-2(c), the formation energies of vacancies were calculated as a function of distance from the interface by minimizing the structure for each vacancy position, corresponding to both interstitial loaded and unloaded $\mathrm{KS}_{\min }$, $\mathrm{KS}_{1}$, and $\{112\}$ interfaces. In Fig. 2(a), for the pristine $\mathrm{KS}_{\min }$ interface, the vacancy formation energy is always positive, but as interstitials are loaded into the interface, sites with negative formation energy appear, which is an indication of spontaneous recombination of those vacancies with interstitials at the interface. As shown in Fig. 2(b), for loaded $\mathrm{KS}_{1}$ interface, more vacancies recombine with interstitials compared to $\mathrm{KS}_{\min }$.

Examination of the recombination process indicates that it begins with an interstitial emitting from the interface, pushing a chain of atoms out from the interface layer to annihilate the vacancy in the bulk. Figure 3 shows the details of one

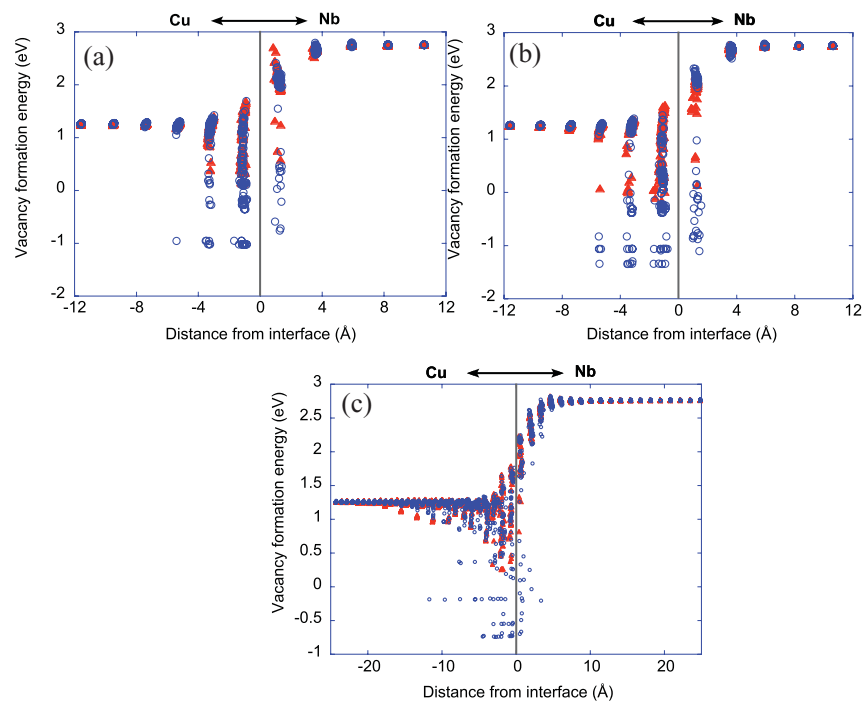

FIG. 2. (Color online) Vacancy formation energies as a function of distance from the interfaces corresponding to both $\mathrm{Cu}$ interstitial loaded and unloaded (a) $\mathrm{KS}_{\min }$ interface, (b) $\mathrm{KS}_{1}$ interface, and (c) $\{112\}$ interface. The open blue circles are for the interstitial loaded interface structures and solid red triangles are for unloaded interface structures. 


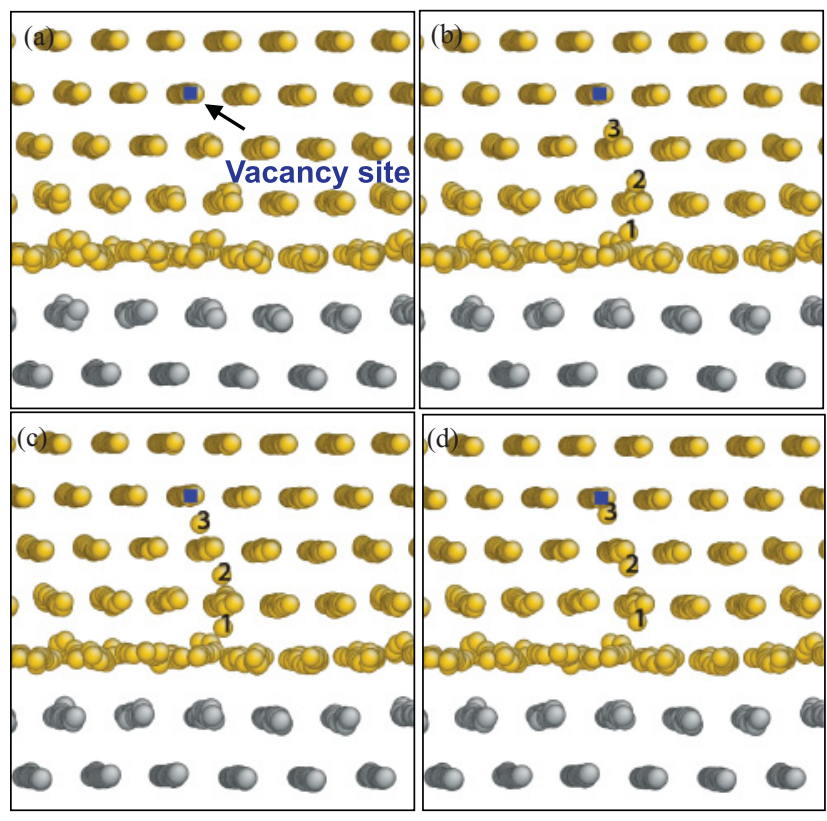

FIG. 3. (Color online) The detailed atomic moves in the observed interstitial emission and recombination event, from NEB calculations. (a) The initial structure, right after the vacancy was introduced away from the interstitial preloaded interface. (b)-(d) The snapshots taken from the process to illustrate how the interstitial atoms emit from the interface and recombine with the vacancy introduced. The smaller (yellow color) atoms are $\mathrm{Cu}$ atoms, and the larger (gray color) atoms are $\mathrm{Nb}$ atoms. The square symbol represents vacancy. The time scale of such a process is estimated to be $\leqslant 0.1 \mu$ s (see text).

such event from the nudged elastic band (NEB) method $^{23,24}$ calculation. In Fig. 3(a), the initial structure is shown, just after the vacancy was introduced about $0.7 \mathrm{~nm}$ away from the loaded interface. Figures 3(b)-3(d) illustrate how an interstitial is emitted from the interface and recombines with the introduced vacancy. The atoms involved in this process are labeled 1-3 in the figure. These three atoms move concertedly, which is analogous to but remarkedly different from the crowdionlike motion of interstitial clusters often seen in irradiated metals. This interstitial emission mechanism is very similar to that found earlier at $\mathrm{Cu}$ grain boundaries. ${ }^{7}$

To understand how the atomic structure of the interface influences the interstitial emission mechanism, a similar loading study was performed on the $\{112\}$ interface. This interface differs qualitatively in that Shockley partials exist on the $\mathrm{Cu}$ side of the interface. ${ }^{18}$ Vacancy formation energies near this interface loaded with $\mathrm{Cu}$ interstitials as well as unloaded are shown in Fig. 2(c). Once the interface is loaded with $\mathrm{Cu}$ interstitials, a number of low vacancy formation energy sites are created, resulting from long-range vacancy-interstitial recombinations. This confirms that the interstitial loading effect is also present on the $\mathrm{Cu}$ side of the interfaces studied here. For all three interfaces studied, sites of reduced $\mathrm{Nb}$ vacancy formation energies are also seen, but limited to only about one layer from interface. In these cases, local intermixing at the interface, a consequence of $\mathrm{Nb}$ vacancies "recombining" with $\mathrm{Cu}$ interstitials at the interface, occurs. Overall, the $\{112\}$ interface has a longer interaction range than the KS interfaces,

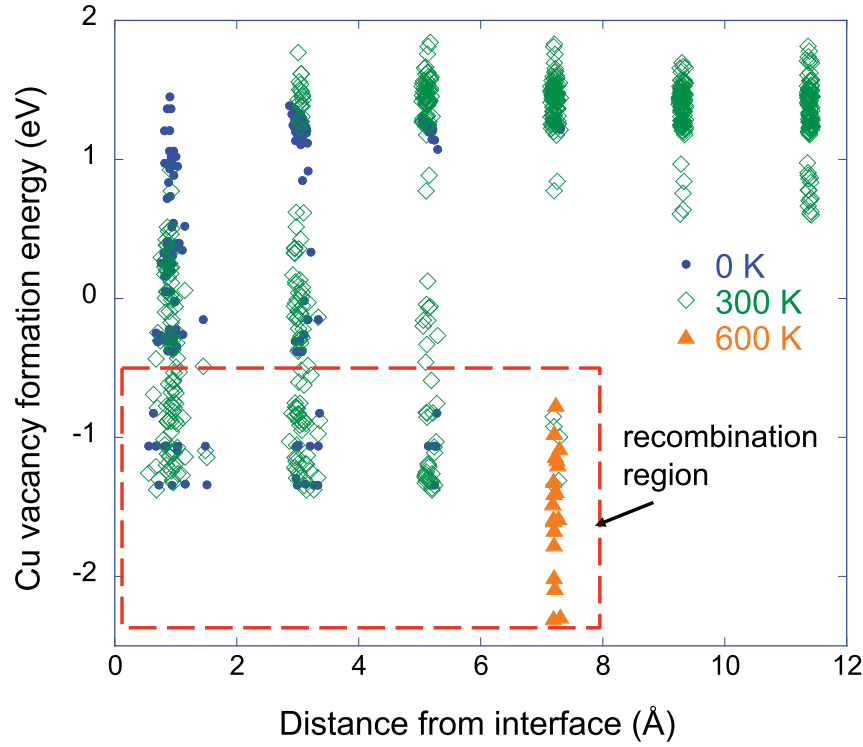

FIG. 4. (Color online) The $\mathrm{Cu}$ vacancy formation energies for $\mathrm{Cu}$ interstitial loaded $\mathrm{KS}_{1}$ interface from energy minimization at $0 \mathrm{~K}$, and $\mathrm{MD}$ at 300 and $600 \mathrm{~K}$, showing increased range and strength of the vacancy-intersitial interaction at higher temperatures as small barrier processes become active. The $600 \mathrm{~K}$ result is obtained from MD for vacancies that were introduced $0.7 \mathrm{~nm}$ away from the interface and only the recombination sites were shown for clarity. A schematic of the recombination region is also shown for guidance purposes.

and slightly higher vacancy formation energetics near the interface.

Returning to the $\mathrm{KS}_{1}$ interface, Fig. 2(b) reveals spontaneous recombination events at $0 \mathrm{~K}$. However, as suggested in Ref. 7, other sites may exist with finite but low barriers for interstitial emission processes. To investigate temperature effects, we begin with the same loaded interface and perform $\mathrm{MD}$ at different temperatures to determine if new recombination events might occur. First, for each vacancy configuration, MD simulations were performed at $300 \mathrm{~K}$ for a period of $10 \mathrm{ps}$. Second, for a subset of vacancy configurations about $0.7 \mathrm{~nm}$ from the interface, a new set of MD simulations at $600 \mathrm{~K}$ for a period of $100 \mathrm{ps}$ were performed. In both cases, the energy of the resulting configuration was minimized. These results are shown in Fig. 4, along with the vacancy formation energy for the loaded $\mathrm{KS}_{1}$ interface at $0 \mathrm{~K}$ [from Fig. 2(b)], for comparison. As the temperature is increased, more vacancies, placed further from the interface, recombine with interstitials at the boundary over longer distances. That is, the effective range and strength of the vacancy-interstitial interaction is increased at higher temperatures as small barrier processes become active. The energies at $600 \mathrm{~K}$ in some cases are markedly lower than all other cases, possibly a consequence of further annealing of the interface.

Finally, we used the NEB method to calculate the transition states and energy barriers for all the events leading to recombinations in $600 \mathrm{~K}$ MD simulations. A total of 21 events were analyzed. There are 20 events (accounting for over $95 \%$ of the total) in which the barriers are less than $0.38 \mathrm{eV}$ [Table S1 (Ref. 25)], which is significantly less than the bulk vacancy migration barrier of $0.68 \mathrm{eV}$, implying that these processes 
would occur much more rapidly than the alternative process of direct vacancy migration to the interface. Based on a simple harmonic approximation, the estimated time for the interstitial emission processes at $300 \mathrm{~K}$ should be $\leqslant 0.1 \mu \mathrm{s}$.

To further test the dependence of our MD simulation results on the interatomic potentials, we performed similar studies using "tunable potentials" by varying the heats of mixing 16,22 of $\mathrm{Cu}-\mathrm{Nb}$ as well as varying the fcc-bcc lattice misfits ratio. ${ }^{26}$ These studies show the same qualitative features as observed in the $\mathrm{Cu}-\mathrm{Nb}$ interfaces, with no exception, although the quantitative details are different.

Earlier, Ackland ${ }^{8}$ raised an issue if the types of processes discussed here and in Ref. 7 would still occur at interfaces where absorbed defects were not "identifiable," or if the interfacial structure was such that defects lost their identity once absorbed. Similarly, as the KS interfaces examined here are nearly perfect sinks in which defects are strongly delocalized, one might have speculated that strong interstitialvacancy interactions facilitated by the interface might not occur for these interfaces. Incidentally, one may also tend to view that when point defects get absorbed at deep traps in grain boundaries or interfaces, these defects may "lose their identity." In such a case, there would be no loading-unloading effect since an interface with interstitials and one without interstitials would be essentially indistinguishable. Our MD simulations show that regardless of the detailed structure of interfaces and the extent of point defect delocalization at interfaces, the interstitial loading-unloading effect exists and that similar mechanism, i.e., the interstitial emission process, occurs. This indicates that interstitials do not lose their identity when absorbed at these interfaces and thus retain the ability to interact with vacancies in the bulk region of the layer.

In summary, MD studies of collision cascades in KS-type $\mathrm{Cu}-\mathrm{Nb}$ interfaces are performed, revealing that $\mathrm{Cu}$ interstitials are preferentially loaded into the interface while on the bcc $\mathrm{Nb}$ side there is very little absorption of interstitials. We study the interstitial loading effect at two types of heterogeneous $\mathrm{Cu}-\mathrm{Nb}$ interfaces, both KS-type and \{112\}-type interfaces. The $\mathrm{Cu}$ interstitials are observed to spontaneously emit from both types of interfaces to annihilate vacancies in the nearby bulk. Further, MD at different temperatures and NEB calculations are used to characterize low barrier emission processes, which promote enhanced recombination near the interface. These results suggest that the self-healing interstitial emission mechanism found at special grain boundaries in single-component $\mathrm{Cu}^{7}$ can be important at heterogeneous interfaces of nanocomposites, which will have interfaces that are more stable under irradiation than single-component materials. Our study also indicates that interstitials do not lose their identity when absorbed even at interfaces where they become significantly delocalized.

The authors thank X.-M. Bai, K. Kolluri, J. Wang, A. J. Caro, and E. M. Saez for insightful discussions. This work was primarily sponsored by the Laboratory Directed Research and Development programs at Los Alamos National Laboratory. The simulations on the $\{112\}$ interface were supported as part of the Center for Materials at Irradiation and Mechanical Extremes, an Energy Frontier Research Center funded by the US Department of Energy, Office of Science, Office of Basic Energy Sciences under Award No. 2008LANL1026.
${ }^{1}$ R. W. Grimes and W. J. Nuttall, Science 329, 799 (2010).

${ }^{2}$ R. W. Grimes, R. J. M. Konings, and L. Edwards, Nature Mater. 7, 683 (2008).

${ }^{3}$ G. R. Odette and D. T. Hoelzer, JOM 62, 84 (2010).

${ }^{4}$ G. R. Odette, M. J. Alinger, and B. D. Wirth, Annu. Rev. Mater. Res. 38, 471 (2008).

${ }^{5}$ F. J. Pérez-Pérez and R. Smith, Nucl. Instrum. Methods Phys. Res. B 164-165, 487 (2000).

${ }^{6}$ M. Samaras, P. M. Derlet, H. Van Swygenhoven, and M. Victoria, Philos. Mag. 83, 3599 (2003).

${ }^{7}$ X. M. Bai, A. F. Voter, R. G. Hoagland, M. Nastasi, and B. P. Uberuaga, Science 327, 1631 (2010).

${ }^{8}$ G. Ackland, Science 327, 1587 (2010).

${ }^{9}$ M. J. Demkowicz, R. G. Hoagland, and J. P. Hirth, Phys. Rev. Lett. 100, 136102 (2008).

${ }^{10}$ T. Hochbauer, A. Misra, K. Hattar, and R. G. Hoagland, J. Appl. Phys. 98, 123516 (2005).

${ }^{11}$ A. Misra, M. J. Demkowicz, X. Zhang, and R. G. Hoagland, JOM 59, 62 (2007).

${ }^{12}$ W. J. Phythian, R. E. Stoller, A. J. E. Foreman, A. F. Calder, and D. J. Bacon, J. Nucl. Mater. 223, 245 (1995).

${ }^{13}$ G. V. Kurdjumov and G. Sachs, Z. Phys. 64, 325 (1930).
${ }^{14}$ P. M. Anderson, J. F. Bingert, A. Misra, and J. P. Hirth, Acta Mater. 51, 6059 (2003).

${ }^{15}$ M. J. Demkowicz, J. Wang, and R. G. Hoagland, in Dislocations in Solids, edited by J. P. Hirth (Elsevier, Amsterdam, 2008), p. 141.

${ }^{16}$ X.-Y. Liu, R. G. Hoagland, J. Wang, T. C. Germann, and A. Misra, Acta Mater. 58, 4549 (2010).

${ }^{17}$ S. B. Lee, J. E. LeDonne, S. C. V. Lim, I. J. Beyerlein, and A. D. Rollett (unpublished).

${ }^{18}$ M. J. Demkowicz and L. Thilly, Acta Mater. 59, 7744 (2011).

${ }^{19}$ M. S. Daw and M. I. Baskes, Phys. Rev. B 29, 6443 (1984).

${ }^{20}$ M. J. Demkowicz and R. G. Hoagland, Int. J. App. Mech. 1, 421 (2009).

${ }^{21}$ J. Wang, R. G. Hoagland, J. P. Hirth, and A. Misra, Acta Mater. 56, 5685 (2008).

${ }^{22}$ J. Wang, R. G. Hoagland, X.-Y. Liu, and A. Misra, Acta Mater. 59, 3164 (2011).

${ }^{23}$ G. Henkelman, B. P. Uberuaga, and H. Jónsson, J. Chem. Phys. 113, 9901 (2000).

${ }^{24}$ G. Henkelman and H. Jónsson, J. Chem. Phys. 113, 9978 (2000).

${ }^{25}$ See Supplemental Material at http://link.aps.org/supplemental/ 10.1103/PhysRevB.85.012103 for Table S1.

${ }^{26}$ X.-Y. Liu, R. G. Hoagland, M. J. Demkowicz, M. Nastasi, and A. Misra, J. Eng. Mater. Technol. (to be published). 